\title{
La chambre dans l'atelier. François Kollar et le travail des artisans (1931-1934)
}

The Chamber in the Workshop. François Kollar and the Work of Craftsmen (1931-1934)

Cédric Perrin

\section{OpenEdition}

\section{Journals}

Édition électronique

URL : https://journals.openedition.org/itti/2483

DOI : $10.4000 /$ itti.2483

Éditeur

Université de Poitiers

Référence électronique

Cédric Perrin, «La chambre dans l'atelier. François Kollar et le travail des artisans (1931-1934)» Images du travail, travail des images [En ligne], 12 | 2022, mis en ligne le 18 février 2022, consulté le 03 avril 2022. URL : http://journals.openedition.org/itti/2483 ; DOI : https://doi.org/10.4000/itti.2483

Ce document a été généré automatiquement le 3 avril 2022.

Images du travail, travail des images 


\title{
La chambre dans l'atelier. François Kollar et le travail des artisans (1931-1934)
}

\author{
The Chamber in the Workshop. François Kollar and the Work of Craftsmen \\ (1931-1934)
}

Cédric Perrin

Je remercie Alain P. Michel et Henri Eckert pour leurs observations sur les versions préliminaires de cet article, la Bibliothèque Forney pour les informations sur le fonds Kollar, et M. Sylvain Besson, directeur des collections du musée Nicéphore Niépce, Ville de Chalon-sur-Saône.

1 "La France travaille », affirment les éditions Horizons de France au fil d'un reportage photographique qu'elles publient en quinze fascicules entre 1931 et 1934. Ce début des années 1930 marque un moment particulier dans l'histoire économique et sociale de la France. Pour la première fois, la population urbaine dépasse la population rurale dans un pays qui se découvre en crise. Ces années sont celles de la Grande Dépression qui suit la crise boursière de 1929. Si en 1930 les dirigeants français pouvaient encore s'imaginer la France comme un îlot préservé, en 1931 le doute n'est plus permis : la crise est bien là et elle frappe durement le pays. La production baisse tandis que le chômage progresse et laisse de nombreux travailleurs sans travail. Les gouvernements successifs répondent par une politique budgétaire orthodoxe et déflationniste qui ne réussit qu'à entretenir la récession et le mécontentement. La crise devient politique et, sous ce jour, atteint son acmé en 1934 quand la manifestation organisée à Paris le 6 février par les mouvements ligueurs d'extrême droite menace, pour une partie de ceux-ci, d'attaquer l'Assemblée nationale et provoque une quinzaine de morts.

2 L'idée de ce reportage revient au directeur artistique d'Horizons de France, le graphiste Maximilien Vox, qui s'est fait connaitre pour ses illustrations de couverture de la maison Grasset ${ }^{1}$. Pour ce projet, il fait appel à un jeune photographe de 27 ans qui vient de s'installer en indépendant : François Kollar. Ferenc, de son prénom de naissance, est né en Autriche-Hongrie en 1904 à Senec (aujourd'hui en Slovaquie) dans une famille 
modeste de langue hongroise (Roegiers, Baqué, 1989 ; Lelieur, Bachollet, 1986). Au cours de son adolescence, il fait ses premières photographies et il commence à apprendre le français durant ses études secondaires à Presbourg (l'actuelle Bratislava) qu'il doit interrompre après la guerre, car, dans la nouvelle Tchécoslovaquie, les cours ne se font plus en hongrois. Il entre alors aux Chemins de fer tchécoslovaques. En 1923, il émigre à Paris et travaille deux ans comme tourneur chez Renault avant de réussir à se faire embaucher comme photographe, notamment chez l'imprimeur Draeger. Il développe son art dans l'exercice de la photographie publicitaire ; ce qui n'est pas sans influencer, on le verra, son œuvre de photographe.

3 Kollar s'investit pleinement dans ce reportage et même au-delà de ce qui était prévu initialement. Pendant quatre ans, il a sillonné le pays, accompagné de son épouse, Fernande Papillon. Les images retenues sont publiées en fascicules thématiques puis rassemblées en deux volumes. Elles sont aujourd'hui conservées par la bibliothèque Forney de la Ville de Paris et librement consultables en ligne ${ }^{2}$. C'est ce fonds Kollar qui a été utilisé pour cette étude et, en conséquence, celle-ci s'est intéressée aux images seules, indépendamment des textes des fascicules des éditions Horizons de France. Cette série de photographies reste, avec celles qu'il réalisa dans la suite des années 1930, son travail le plus connu. En 1940, Kollar qui refusait de travailler avec le nouveau régime s'installa à Poitiers, à la différence de Maximilien Vox qui participa aux activités de propagande de Vichy en lançant le nouveau magazine Vrai, en 1941, par exemple. Il reprit son activité de photographe à Paris en 1945, mais sans retrouver sa notoriété d'avant-guerre.

4 La démarche des éditions Horizons de France n'est ni inédite, ni isolée. Elle fait écho, par exemple, à la vaste enquête photographique Les Archives de la planète, financée par le banquier et mécène Albert Kahn entre 1909 et 1931, ou à celle réalisée, entre autres, par Dorothea Lange, Paul Taylor ou Walker Evans pour l'agence fédérale états-unienne de la Resettlement Administration créée par Roosevelt en 1935. Le reportage commandé à Kollar concerne plus précisément le travail, c'est-à-dire pour les économistes, les activités productives qui créent de la valeur (ce qui exclut le travail domestique ou scolaire notamment). C'est bien en effet à un vaste tableau en images de la production française qu'il aboutit. Il n'est pas le premier à photographier le travail dans l'Europe de l'entre-deux-guerres (Peroni, Roux, 1996; Chevandier, 2004). Le mouvement de la photographie ouvrière connut un grand essor en Allemagne entre 1926 et 1932 autour de la revue Der Arbeiter-Fotograf, mais aussi en Angleterre, aux Pays-Bas, aux États-Unis, en Belgique ou encore en Tchécoslovaquie et en URSS (Joschke, 2020). François Kollar a été ouvrier à son arrivée à Paris, mais il ne l'est plus dans les années 1930. Il ne peut pas être rattaché à ce mouvement, mais certaines évolutions esthétiques que connaît celui-ci à la fin des années 1920 se retrouvent, comme on le verra, dans sa façon de photographier artisans et ouvriers. Il est de la génération des Henri Cartier-Bresson, Willy Ronis ou Robert Doisneau (nés respectivement en 1908, 1910 et 1912) qui débutèrent également dans les années 1930 ; il demeure cependant un « observateur plus lointain » (Albera, 2018) du mouvement de la photographie sociale (ou humaniste, mais cet adjectif est désormais critiqué par les historiens de la photographie). Il n'est pas un photographe engagé. Il ne s'agit pas pour lui de dénoncer des injustices. Il photographie ces travailleurs et travailleuses sur leur lieu de travail et s'attarde rarement sur leurs conditions de vie. Pour autant, François 
Kollar met en images une France populaire. Cette France qui travaille est celle des ouvriers, mineurs, paysans, marins et artisans.

Ce sont à ces derniers que cet article s'intéresse alors que des publications ont déjà été consacrées aux paysans (Kollar, 2010), cheminots (Kollar, 1986) et pêcheurs (CalameLevert, Decoux, Perroy, 2012). La démarche croise ces photographies avec d'autres documents et l'état des connaissances historiques sur les artisans. En confrontant ces matériaux, il est alors possible de repérer les écarts et les concordances. Une image porte «les marques du regard» de celui qui les a créées et elle engage des valeurs: " montrer, c'est d'abord choisir, sélectionner ce qui sera vu et, donc, ce qui ne sera pas vu» (Terrenoire, 1985). Qu'est-ce que les photographies de Kollar nous apprennent, confirment ou illustrent au sujet du travail des artisans ? Et, à l'inverse, quelle image des artisans a construite François Kollar en posant sa chambre noire dans leurs ateliers? Qu'a-t-il voulu nous en montrer? Qu'est-ce qui l'a intéressé et, au contraire, qu'a-t-il laissé de côté ou n'a pas vu? Dans quelle mesure le reportage photographique est-il une archive des artisans?

\section{Un artisanat en mouvement au miroir du reportage photographique}

6 Pour répondre à ces questions, il paraît nécessaire de prendre d'abord la mesure, même à grands traits ici, de l'artisanat dans les années 1930. Sur près de 2000 photographies de $\mathrm{La}$ France travaille, une cinquantaine le concerne, soit une assez faible proportion (environ $3 \%$ ). Il est difficile d'établir un comptage très précis, car ni les photographies ni leurs légendes ne permettent toujours de déterminer le statut de celui ou celle qui est photographié. Sans surprise, les artisans sont totalement absents de plusieurs séries (mines, aviation, rail...). Ces industries se sont constituées hors de l'artisanat. Il ne faut pas y voir l'effet d'une concurrence industrielle qui alimenterait un déclin de ce dernier. La Confédération générale de l'artisanat français revendiquait dans les années 1920 le chiffre de 800000 artisans. Le registre des métiers, ouvert en 1936, permet d'estimer que ceux-ci étaient environ 750000 à l'issue de la Grande Dépression (Perrin, 2007). L'artisanat était loin d'être un monde en déclin profond dans l'entredeux-guerres.

\subsection{Un monde diversifié et en mutation}

7 Les artisans sont très présents dans trois secteurs d'activité qui concentrent à eux seuls plus de la moitié d'entre eux dans les années 1930 : le bâtiment, qui représente plus d'un quart du total, l'alimentation et la confection qui en rassemblent 15 à $20 \%$ chacun (Perrin, 2007). Le bâtiment englobe une grande variété de corps de métiers (maçon, charpentier, couvreur, peintre, menuisier, plombier...) qui ont conservé une structure artisanale, et où les grandes entreprises restent peu nombreuses. L'alimentation comprend notamment la boulangerie-pâtisserie et la boucherie-charcuterie, mais compte aussi, en plus petits nombres, des producteurs d'huile, de bière ou de biscuits par exemple. Dans le textile, les artisans tisserands ont subi précocement l'industrialisation et ne subsistent de façon résiduelle que sur des niches et en quelques lieux, mais les artisans demeurent très nombreux dans les métiers de la confection (couturière, tailleur, fourreur, modiste...). 
Ces trois piliers sont inégalement représentés dans La France travaille. Les images du fascicule dédié au bâtiment ont été faites en région parisienne sur des chantiers de moyennes ou de grandes entreprises, comme Sainrapt et Brice, si bien que peu d'artisans sont présents : l'atelier d'un menuisier et deux jeunes maçons réparant un mur. Au regard de leur importance tant dans l'artisanat d'une part que dans le bâtiment d'autre part, ces artisans sont nettement sous-représentés. Cela peut s'expliquer par une question de méthode. Les photographies n'ont pas été faites au hasard de déambulations urbaines, mais en suivant un programme préétabli de visites d'entreprises pour lequel des contacts ont été pris par l'éditeur avec de moyens ou grands établissements (Chevrier, 2016). L'alimentation est beaucoup mieux illustrée avec notamment deux séries, l'une dans le fournil d'un boulanger parisien, une autre dans une huilerie en Provence. La boucherie, par contre, ne s'y trouve qu'avec un artisan en tournée dans les Charentes; les images de garçons bouchers des Halles déchargeant, découpant et pesant des carcasses renvoient au commerce de gros plutôt qu'à l'artisanat.

9 Les images du textile sont plus ambiguës et en composent un tableau un peu déformé, pour deux raisons notamment. D'une part, François Kollar s'est focalisé sur des métiers en déclin ou marginaux (tisserands, brodeuses, dentellières) alors que couturières et tailleurs sont absents. Des couturières sont bien montrées, mais ce sont des ouvrières, les «petites mains» des maisons de mode de Paris (celle des sœurs Callot, par exemple), qui figurent par ailleurs dans la clientèle habituelle de François Kollar et dans lesquelles il devait donc avoir ses entrées (Denoyelle, 1995). Une modiste parisienne apparaît de façon presque anecdotique parmi les métiers du fer, travaillant un fil de fer pour monter les ornements d'une coiffe ${ }^{3}$. D'autre part, une partie de ceux qui sont présentés comme des artisans n'en sont probablement pas. Deux indices le laissent deviner dans la série des tisserands picards : les légendes indiquent le nom de la société Rodier de Bohain-en-Vermandois; une autre photographie prise dans cette même société montre les « voitures rapportant à la fabrique les tissus "4. Ces tisserands sont donc plus certainement des ouvriers à domicile, des façonniers, qui travaillent pour l'entreprise Rodier qui distribue du travail dans les villages voisins puis récupère les draps tissés. 
Image 1 : Poterie de la Côte d'Azur

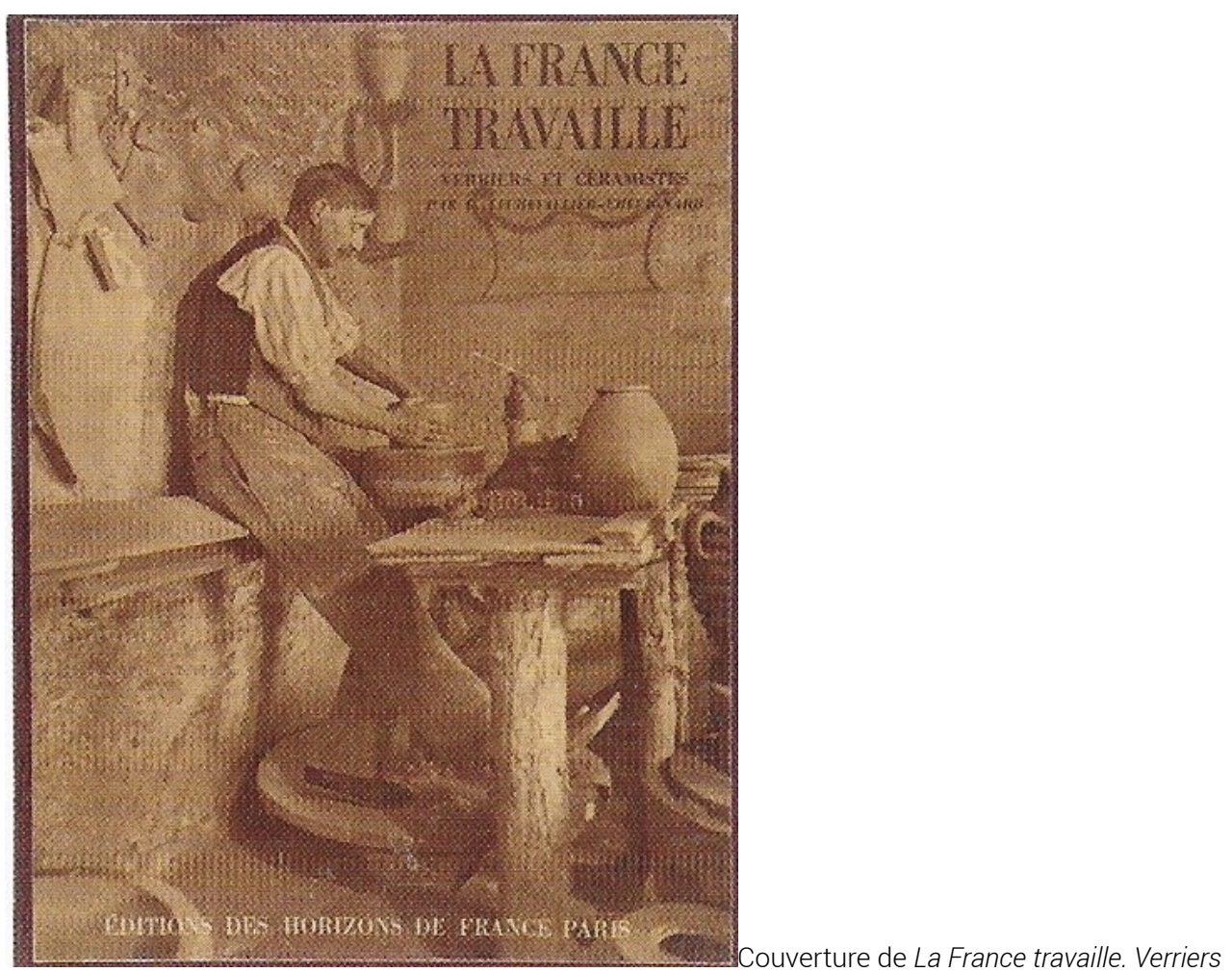

et céramistes, vol. 12, Paris, 1934. du bois (ébéniste, tonnelier...), des métaux, du cuir (cordonniers, tanneurs, bourreliers...) et les industries diverses (imprimerie, verre, céramique...) - qui ne rassemblent chacune qu'une petite part (autour de $5 \%$ ) des artisans. On y trouve des métiers anciens, pour partie en déclin, plus exposés à la concurrence de l'industrie, mais celle-ci ne suffit pas à expliquer leurs dynamiques; l'évolution des marchés, des matériaux, des usages, de la demande, est aussi à prendre en considération. Ils sont peu présents dans le reportage : une ou deux photographies pour le bois et le fer, aucune pour le cuir. La poterie, pourtant très secondaire, a les honneurs d'une petite série et de la couverture du fascicule "Verriers et céramistes" (image 1). Ces métiers se caractérisent aussi par une grande dispersion géographique couplée à une concentration spatiale dans des systèmes productifs localisés, ou districts industriels, qui réunissent dans un même lieu des grappes de petites et moyennes entreprises et d'artisans d'une même activité : chaussures à Fougères ou à Romans, tanneries à Château-Renault, couteliers de Thiers..., des lieux dans lesquels Kollar ne s'est pas rendu.

11 À côté de ces activités de production, les services sont en plein essor. Les branches de l'entretien de la personne (coiffure notamment) et des vêtements (lingère, blanchisseuse, teinturerie...) et celle du dépannage et de la réparation émergent et rassemblent déjà chacune 10 à $12 \%$ des artisans, auxquels s'ajoutent diverses activités de services moins développées comme les transports (taxi, camionneur, marinier...) et divers nouveaux métiers dont celui de photographe. La réparation et le dépannage sont bien documentés avec plusieurs mécaniciens automobiles, de même que les transports,

Images du travail, travail des images, 12 | 2022 
avec notamment le fascicule "Mariniers, bateliers", alors que les taxis, coiffeurs ou coiffeuses, blanchisseuses ou teinturiers restent absents.

Le reportage photographique de François Kollar n'est pas totalement incohérent avec ce panorama, mais sans y être vraiment fidèle. Il présente quelques écarts notables avec des métiers surreprésentés alors que des absences laissent tout un pan des activités artisanales hors champ. Il s'attarde sur quelques vieux métiers sur le déclin sans oublier toutefois les nouveaux, dans une alternance de nostalgie et de modernité qui se retrouve dans l'ensemble de La France travaille (Roegiers, Baqué, 1989 ; Chevrier, 2016).

\subsection{L'émergence d'un groupe socioprofessionnel}

Les artisans que photographie François Kollar composent un groupe socioprofessionnel qui est en cours de structuration dans l'entre-deux-guerres. Cette organisation est source de débats et de tensions, notamment autour de ce que sont un artisan et l'artisanat.

Selon la racine latine ars, l'artisan est un homme de l'art (des arts mécaniques par opposition aux arts libéraux), l'homme de métier, c'est-à-dire l'ouvrier (au sens premier de celui qui œuvre) qui maîtrise les techniques de son travail. Le mot est entré dans la langue française depuis l'italien au XvI ${ }^{\mathrm{e}}$ siècle (Rey, 1992 ; Perrin, 2020), et les dictionnaires du début du XIX ${ }^{e}$ siècle lui conservent cette définition. C'est cet emploi qui se retrouve dans les légendes des photographies qui font des tisserands picards et passementiers stéphanois des « artisans » alors que ce sont plus vraisemblablement des ouvriers à domicile; tout comme il reste d'usage actuellement de parler d'artisans pour désigner les ouvriers très qualifiés des entreprises du luxe par exemple. Dans les années 1930, il s'agissait toutefois d'un usage déjà vieilli. En effet, au cours du $\mathrm{XIX}^{\mathrm{e}}$ siècle, avec l'industrialisation, les artisans se sont détachés du groupe des ouvriers d'industrie pour former un groupe social intermédiaire, une classe moyenne, entre ceux-ci et les patrons (Perrin, 2020). L'artisan est devenu l'ouvrier qui travaille pour son propre compte, un travailleur indépendant, voire déjà un entrepreneur ou un petit patron. Et ce sont bien, on le verra, des ouvriers à leur compte que nous montre François Kollar.

La (re)définition se précise durant l'entre-deux-guerres alors que l'artisanat prend corps et s'organise. Le terme "artisanat" apparaît dans le vocabulaire français au lendemain seulement de la première guerre mondiale et il est donc encore relativement neuf au début des années 1930. En 1918, la France a récupéré l'AlsaceLorraine où les artisans disposaient déjà d'une Chambre de métiers, en application d'une loi allemande de 1897. Les dirigeants de la Chambre de métiers d'Alsace se sont alors employés à défendre leur statut et à le proposer en modèle à introduire en France. Dans le même temps, un groupe de cordonniers parisiens emmenés par Robert Tailledet et Georges Grandadam, encouragés par le sénateur radical Étienne Clémentel, a entrepris de fédérer les artisans (Kessler et Rousseau, 2018). Les deux courants se réunirent pour fonder la Confédération générale de l'artisanat français (CGAF) à Strasbourg en 1922. Ainsi organisés, les artisans français obtiennent un statut juridique particulier avec l'adoption dans les années 1920 des lois sur leur statut fiscal, la création du crédit artisanal puis des Chambres des métiers. Toutefois, en désaccord sur 
la stratégie et la définition de l'artisan, les Alsaciens quittent rapidement la CGAF et l'artisanat se divise (Zarca, 1986 ; Zdatny, 1999).

16 La controverse rebondit au début des années 1930 - quand Kollar réalise son reportage - à l'occasion des débats qui accompagnent la proposition de créer un registre des métiers, l'équivalent d'un registre du commerce spécifique aux entreprises artisanales. La question se pose alors de savoir qui devra s'y inscrire, ce qui revient à définir qui est artisan. La CGAF défend l'introduction d'un seuil de main-d'œuvre, car, pour ses représentants, l'artisan participe habituellement et personnellement à la production avec ses ouvriers, s'il en emploie (Tailledet, 1937). C'est d'abord un petit travailleur indépendant des professions dites manuelles. Or, au-delà d'une certaine taille, ce n'est plus le cas, car l'entrepreneur se concentre alors sur la direction de l'entreprise. Les dirigeants alsaciens, qui sont eux-mêmes des petits patrons qui emploient plus d'une vingtaine de salariés, ne veulent pas entendre parler de cette limite qu'ils jugent arbitraire et "mathématique» (Daty, 1933); pour eux, seule la qualification fait l'artisan. La loi votée en 1934 retient finalement un seuil de cinq salariés, et le registre des métiers entre en service en 1936 ; les départements alsaciens conservant le bénéfice de leur statut antérieur sans cette limite. À cet égard, même s'il est difficile de dire s'il s'agit d'une intention délibérée, il n'est pas anodin que Kollar ne se soit pas rendu en Alsace et qu'il n'ait pas non plus photographié de cordonniers. Comme cela a pu lui être reproché sur d'autres questions sociales, il ne s'engage pas, ne prend pas parti et esquive ce débat.

\subsection{Un artisan du regard}

17 On ne sait pas ce que François Kollar connaissait précisément de ces débats professionnels. Quelques indices laissent toutefois penser qu'ils ne pouvaient pas lui être totalement inconnus. Son métier et son parcours le rapprochent du monde des artisans. Ils font de lui un artisan du regard, un «ouvrier du regard» indépendant (Collectif, 2016).

18 Ces débats sont ainsi rapportés dans la revue professionnelle Le Photographe. Le journal, dirigé par le Tourangeau Paul Montel, fondateur de l'École technique de photographie et de cinéma en $1926^{5}$, se veut l'organe des photographes français professionnels et se réserve d'ailleurs le droit de refuser un abonnement «aux personnes n'exerçant pas une profession en rapport avec la photographie». Sa rédaction considère que «la profession de photographe compte une proportion importante d'artisans » et elle publie régulièrement "une documentation fort utile » à ceux-ci. En février 1933, par exemple, elle reproduit in-extenso sur six pages un projet de loi sur l'artisanat ${ }^{6}$ avec son exposé des motifs qui revient sur "les différentes définitions »" Il est certain que «François Kollar, le photographe bien connu » connaissait la publication puisqu'il y a signé, par exemple, une publicité pour les lampes Philips en 1935, illustrée d'ailleurs par l'une des photographies de La France travaille ${ }^{8}$. Il y est cité, au moins, à quatorze reprises entre 1934 et 1938. Il ne paraît donc pas déraisonnable de penser qu'il était informé de la situation des artisans par la lecture de ce journal.

19 Le parcours professionnel de Kollar suit celui de la majorité des artisans. Comme on l'a $\mathrm{vu}$, après avoir travaillé quelques années comme salarié, d'abord chez Renault, puis comme photographe dans plusieurs imprimeries, il s'installe à son propre compte alors qu'il est encore assez jeune. Il n'a que 26 ans quand il crée son studio et se fait inscrire 
au registre du commerce de Paris en 1930. À titre de comparaison, un tiers des artisans qui se sont inscrits au registre des métiers en 1938 avaient moins de 30 ans (Perrin, 2007). Comme la plupart des artisans également, il s'installe avec peu de moyens et il travaille seul, sans salarié. C'est son épouse, voire lui-même, qui lui sert de modèle à ses débuts (Roegiers, Baqué, 1989; Denoyelle, 1995). Il suit ainsi un parcours classique d'artisan. Comme ses confrères photographes - dont au demeurant le quotidien était plus fait de photographies de naissance, de mariage, en entreprise ou de portraits que d'expériences artistiques -, il a dû s'inscrire au registre des métiers à l'ouverture de celui-ci'. Par ailleurs, la distance qu'il conserve à l'égard du mouvement ouvrier, contrairement à d'autres photographes politiquement plus engagés, peut s'interpréter comme celle d'un travailleur indépendant, d'un artisan. Il fréquente peu les milieux artistiques de son temps et reste d'abord un praticien et un homme de métier.

\section{La photographie en archive des artisans}

La publication est guidée par quelques contraintes à la fois matérielles et éditoriales. L'éditeur l'oriente à travers le programme des entreprises visitées et une ligne où se mêlent régionalisme et nostalgie, progressisme et patriotisme industriel dans la promotion du travail, de la maîtrise technique et du savoir-faire d'une France moderne et inventive (Collectif, 2016). François Kollar suit la commande tout en la sublimant grâce à son talent. La valeur de ses photographies d'artisans, comme de l'ensemble, tient d'abord à leur grande qualité esthétique ${ }^{10}$. Kollar maitrise les effets de lumière, joue sur les contrastes, s'appuie sur les lignes pour construire ses images, alterne profondeur de champ et cadrage serré, crée des synecdoques photographiques en ne montrant qu'une partie pour mieux suggérer le tout. Les compositions sont particulièrement soignées. Dans une période durant laquelle les échanges et les emprunts sont importants entre photographies publicitaire et artistique, Kollar a également appris dans la pratique de la première à travailler ses images pour magnifier leur sujet. La photographie publicitaire répond à une commande et mène vers des images gratifiantes et valorisantes et donne une vision positive des conditions de travail. La représentation du travail par les éditions Paul-Martial, par exemple, s'organise autour de cinq types : la figuration, l'épopée, l'absorbement, la théâtralité et la pédagogie (Callens, 2020) qui se retrouvent, en partie, dans les photographies des artisans de La France travaille ${ }^{11}$. Celles-ci composent en effet un ensemble tout à la fois épique et lyrique et documentaire et didactique, qui rapproche le travail des artisans de celui des ouvriers, tout en laissant certains aspects hors champ.

\subsection{Un travail valorisant et porteur de sens}

21 La touche du photographe publicitaire se retrouve dans les images d'artisans dont il photographie, d'une part, le travail mais aussi, d'autre part, le fruit de ce travail, les produits. François Kollar met en valeur, magnifie les artisans. Il s'intéresse aux acteurs du travail, les travailleurs et les travailleuses, avant de photographier ce qu'ils font, le travail lui-même. S'il ne peut pas être rattaché au courant dit de la photographie humaniste qui ne s'est pleinement déployé qu'après la guerre, certains, comme l'historienne de la photographie Françoise Denoyelle, ont vu dans cette attention aux hommes un certain « humanisme photographique » (Roegiers, Baqué, 1989 ; Denoyelle, 
1995). En 1934, le critique d'art Jacques Guenne comparait La France travaille à la littérature de Zola pour en souligner le ton « profondément humain » (Guenne, 1934).

Le travail de l'artisan est montré comme une source de fierté. Il est valorisant et il est valorisé par la photographie. Cette dimension se retrouve plus particulièrement dans les portraits. Ceux-ci sont très différents des portraits d'artisans des Hommes du $\mathrm{XX}^{e}$ siècle, très statiques, réalisés quelques années plus tôt par l'Allemand August Sanders, partisan d'une photographie exacte. Marchés. Type de boulanger, par exemple, présente l'artisan en contre-plongée, torse nu et les bras croisés, le regard fixé vers nous $^{12}$. La lumière le détache de l'arrière-plan où se dessine son ombre sous les lignes des panetons rangés en hauteur. L'image exprime la satisfaction du travail fini et la vigueur physique $\mathrm{du}$ travailleur. Les qualités intrinsèques de ces portraits minutieusement construits font leur expressivité. La force des images évoque métaphoriquement celle des travailleurs. Il a souvent été reproché à Kollar de ne pas dénoncer la dureté des conditions de travail. Dès 1934, Jacques Guenne indiquait, mezzo voce, sans vraiment critiquer, qu'« il n'insiste jamais sur le tragique de destinées ». Ces critiques sont fondées mais par ailleurs, ces portraits s'inscrivent dans une évolution de la photographie ouvrière, sensible notamment en Allemagne à la fin des années 1920, qui s'éloigne du naturalisme et du misérabilisme pour préférer " une fierté incarnée par les signes de la vigueur physique, la vue en contre-plongée et les vêtements propres » (Joschke, 2020). Au cours des années 1930, la revue communiste Regards a proposé le même type d'images. Il est toutefois à souligner qu'avec la série des Éditions de France, elles servent une idéologie traditionaliste et bien différente, notamment sous la plume de Pierre Hamp, l'un de ses auteurs les plus prolixes.

Une autre limite, moins souvent soulignée, tient à la démarche de l'auteur. Ces portraits s'inscrivent dans une collecte de types de travailleurs. Par exemple : Métiers du Fer. Type de fondeur ; Dans le port, à bord. Type de marin ou encore Marchés. Type d'ouvrier cultivateur. Mais la typologie conduit à des images qui ont beaucoup de similitudes, avec les mêmes cadrages, l'usage de la contre-plongée, les bras croisés ou les mains posées sur les hanches, le regard fixe... Type de boulanger n'est pas sans rappeler le Porteur de rails, souvent cité et reproduit (Denoyelle, 1995, p.15, par exemple). Artisans et ouvriers, mais aussi paysans et pêcheurs, y prennent les mêmes attitudes. Outre que ces portraits font l'impasse sur les conditions de travail, la répétition du traitement leur enlève une partie de leur valeur informative et documentaire. La volonté de documenter le travail fait que les types y deviennent des stéréotypes (Morel, 2001 ${ }^{13}$ ) et les images des clichés.

Kollar photographie des artisans et leur travail mais aussi le produit de ce travail, les objets réalisés. C'est un travail qui a une finalité et un sens. Plusieurs vues s'arrêtent ainsi sur les productions de ces artisans : des pains, des madeleines, des pots culinaires, des tonneaux... Elles sont photographiées en plan rapproché, sans profondeur. Ces images d'objets décontextualisées font écho à celles d'Emmanuel Sougez et de la Nouvelle Objectivité, mais elles s'en éloignent par leur suggestivité qui, là aussi, porte la marque de la photographie publicitaire. Dans Le Panier de pains, par exemple, ces derniers prennent chair grâce à une composition qui soulignent leurs formes et le grain de la matière, qui laisse deviner la mie sous la croûte ${ }^{14}$.

Les séries de photographies réalisées chez un même artisan permettent de décomposer la fabrication de ces produits. Elles illustrent le concept de chaîne opératoire d'André Leroi-Gourhan. Chaque image s'arrête sur un maillon de la chaîne. Par exemple, les sept 
vues de l'huilerie d'Aimé Doussant (image 2) présentent dans leur succession quelquesunes des opérations de la transformation des olives en huile: première, deuxième, troisième opérations, pressage, sous-tirage, transvasement. Pour le boulanger et la fabrication du pain: le pétrissage, le façonnage des baguettes, le repos dans les panetons, l'entaille de la pâte, l'enfournage et enfin les pains cuits. L'image sériée est un dispositif didactique qui vise à " expliquer » aux lecteurs des fascicules comment un artisan produit son huile ou son pain, mais elle présente aussi un travail non parcellisé dont l'artisan réalise lui-même les différentes phases de la chaîne opératoire. C'est la même personne qui apparaît sur chaque image, à chaque étape. La production artisanale incarne une culture, voire une éthique, du travail (Sennett, 2010) qui contraste avec le «travail en miettes» de l'industrie fordiste (Friedmann, 1956) - qui commence à se diffuser dans la France de l'entre-deux-guerres et que Kollar lui-même a pu éprouver dans les ateliers des usines Renault - qui dépossède l'ouvrier de la finalité de ce qu'il fait.

Image 2 : L'huilerie d'Aimé Doussant

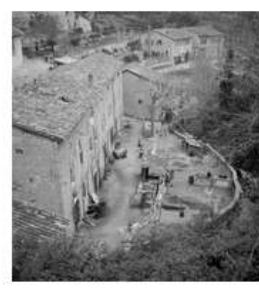

La vie paysanne. Huilerie
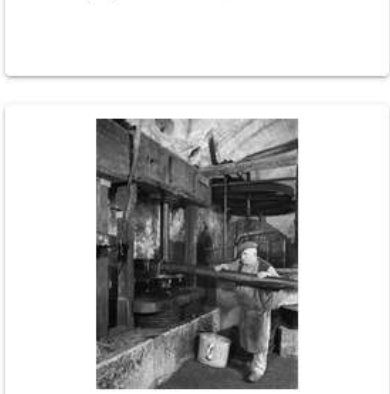

La vie paysanne. Huilerie. Le pressoir à olives

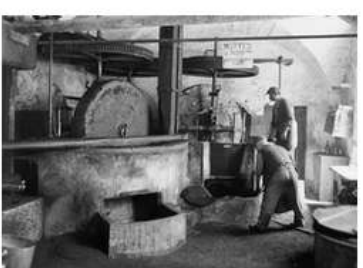

La vie paysanne. Huilerie. 1ere opération

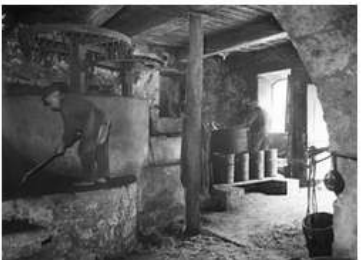

La vie paysanne. Huilerie. 3eme opération

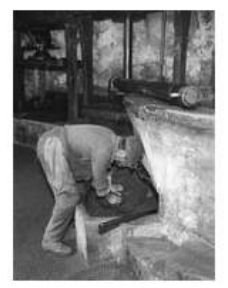

La vie paysanne. Huilerie. 2eme opération

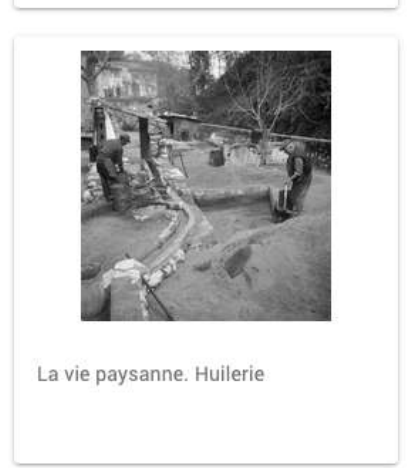

Source : capture d'écran des vignettes des photographies de la série K La Vie paysanne sur le portail de la bibliothèque Forney. https://bibliotheques-specialisees. paris.fr/ark:/73873/pf0000618362.locale=fr

\subsection{Une didactique des corps au travail}

Quand il se lance dans cette commande, François Kollar travaille à la chambre sur plaques de verre de grand format, un matériel lourd et encombrant. Sa chambre GillesFaller pèse près de quatre kilogrammes. Elle suppose l'emploi d'un trépied et de longs temps d'exposition, sans oublier le matériel d'éclairage. Ces contraintes techniques ont des conséquences sur la façon de travailler du photographe qu'il ne faut pas imaginer se fondant dans l'atelier prêt à saisir l'action sur le vif, le travail en train de se faire. Les photographies sont posées. Les artisans reproduisent, ou plutôt suspendent, devant Kollar, les gestes de leur travail, ce qui contribue à conférer au reportage son style 
documentaire et pédagogique. Au cours du reportage, Kollar acquiert un Rolleiflex, un boîtier plus léger de la nouvelle génération qui arrive sur le marché, qui lui donne plus de mobilité et lui permet d'adopter des angles de vue moins convenus, comme la contre-plongée. Pour autant, il ne modifie pas radicalement sa façon de photographier les hommes au travail qui, au-delà des facteurs techniques, relève donc bien aussi d'un choix. Il n'abandonne pas non plus sa chambre avec laquelle il réalise un peu plus de la moitié des vues de La France travaille (Collectif, 2016).

La portée pédagogique se retrouve dans l'attention aux gestes et aux postures du travail. La focalisation sur ceux-ci a des limites bien identifiées. On peut s'interroger sur ce que signifie cette concentration de l'attention sur la réalisation du geste technique et ce qu'elle peut masquer (Deilhes, 2016). De même, les cadrages serrés sur les mains dépersonnalisent l'action technique dans laquelle celles-ci sont réduites à de simples outils, même s'ils ont aussi une finalité pédagogique en montrant un savoirfaire (Callens, 2020). Toutefois, Kollar utilise peu ce type de cadrage. Ces artisans ont un visage. Les corps ne sont pas anonymes. De plus, si ces images des gestes du travail ne disent pas grand-chose sur la condition sociale des travailleurs, elles sont intéressantes pour elles-mêmes. Les gestes et les corps sont au cœur de la démarche anthropologique qui a permis de renouveler la réflexion historique sur le travail des artisans et leurs productions, et que la photographie vient documenter et illustrer.

L'attention aux postures et aux gestes productifs montre comment les corps sont en jeu dans le travail des artisans. Celui-ci s'appuie sur des savoir-faire corporels. Il demande l'acquisition de coups de main, de coups d'œil ou encore d'avoir l'oreille. Le Petit mécano de village a ainsi la tête plongée sous le capot ouvert de la voiture ${ }^{15}$. Il est à l'écoute du moteur pour en repérer les ratés et identifier à l'oreille la nature de la panne avant d'intervenir. Son diagnostic repose sur la mobilisation de son audition, sur une compétence sensitive. C'est au toucher que le menuisier conduit son ciseau, sent les éventuels défauts à corriger sur la pièce de bois qu'il travaille et en apprécie la finition ${ }^{16}$. En ne cadrant pas uniquement sur les mains, Kollar montre aussi l'importance de la vue et de la coordination des gestes. Le Tonnelier du village suit du regard le marteau dans une main qui s'abat sur le coin tenu de l'autre main ${ }^{17}$. Ces savoir-faire corporels sont aussi des savoir-faire incorporés. La réalisation de ces gestes techniques a été intégrée par le corps de l'artisan. Elle repose sur l'apprentissage et l'expérience. Ce sont des gestes acquis par la répétition et que le corps reproduit sans avoir besoin d'y réfléchir et de les décomposer mentalement. L'artisan les a incorporés. Il en a fait des «routines" (Schwint, $2002 \& 2005$ ). La précision et la qualité de son travail dépendent de leur maitrise. Kollar montre ici un critère premier de la définition de l'artisan : la maîtrise de son art, l'habileté de l'homme de métier. Ces artisans sont des ouvriers qualifiés qui maîtrisent les techniques de leur métier. Ces photographies donnent à voir ce que l'ingénieur et photographe Christian Huret décrit comme «la "banale" perfection des gestes de l'artisan ou de l'ouvrier" dans le "spectacle ordinaire du travail » où « le corps est en jeu » (Huret, 2014).

En revanche, les risques que prend l'artisan en mettant ainsi son corps en jeu sont éludés. Le traitement photographique prend le parti de valoriser le travail, et ainsi l'habileté et la force physique éclipsent la pénibilité. Pas plus qu'avec les ouvriers, et bien que les artisans soient leur propre patron désamorce la critique sociale, la dureté du travail n'est montrée. Pourtant, la répétition des gestes et les charges lourdes éprouvent les corps des artisans en causant des troubles musculo-squelettiques 
(Crasset, 2017). Les dangers du travail en hauteur, des matières toxiques et des poussières inhalées, des outils et des machines qui peuvent blesser, voire mutiler, la fréquence des phalanges coupées parmi les menuisiers, par exemple, restent aussi hors champs. Ces photographies ne disent rien non plus des longues heures de travail que les artisans s'imposent chaque semaine pour un revenu à peine plus élevé que celui d'un ouvrier (Perrin, 2007).

Le jeu de l'analogie visuelle rapproche les artisans du monde ouvrier, y compris en creux, dans ses ellipses. Artisans et ouvriers réalisent les mêmes gestes, avec les mêmes risques. Ils partagent des savoir-faire de métiers. Ils ont en commun une culture technique. Mais alors que l'ouvrier œuvre pour un patron qui l'emploie, l'artisan est son propre patron et exerce ses compétences pour lui-même. Ainsi, quand François Kollar les photographie dans les années 1930, les artisans sont encore pour l'essentiel des ouvriers qui se sont mis à leur compte, qui travaillent pour eux-mêmes. Jusqu'au milieu du xxe siècle, la majorité travaille seul, sans salarié (Perrin, 2007) comme la quasi-totalité de ceux du reportage. En outre, ceux qui emploient un ouvrier, comme le menuisier, continuent de travailler avec lui. L'artisan participe personnellement à la production. Il met la main à l'ouvrage.

Dans cette exposition des corps au travail, une attention particulière est accordée aux mains. Elle renvoie à l'idée couramment admise que l'artisan est un travailleur manuel. Kollar se montre d'ailleurs sensible à cette tradition. Invité en 1936 à présenter une de ses photographies dans les pages du magazine $V U$, un hebdomadaire d'informations illustré, il choisit celle d'un luthier en expliquant, dans la légende : «Je la préfère parce que je trouve admirable l'effort persévérant de l'artisanat qui, par amour de son travail et de son métier, a encore le courage et l'espoir de survivre à la machine » (image 3).

Image 3 : François Kollar, La Mise en place de l'âme

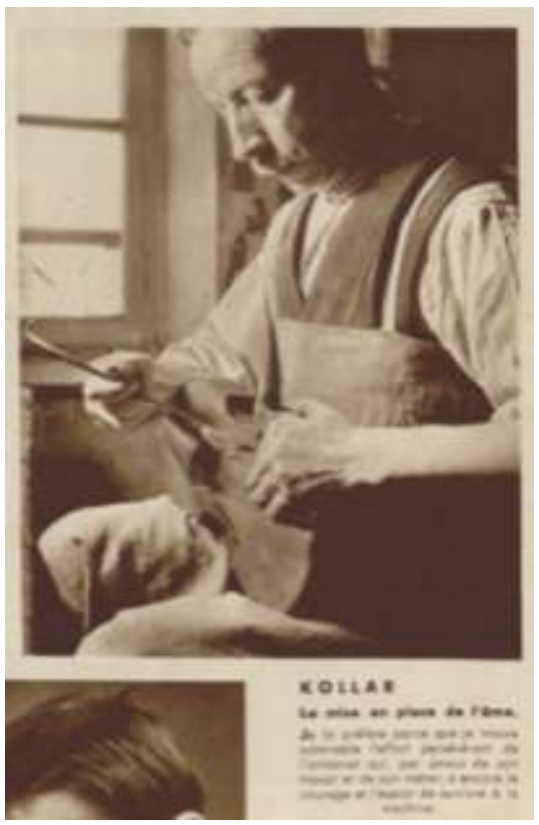

Source : VU, nº 422, 15 juillet 1936, p. 14.

Crédit : Musée Nicéphore Niépce, Ville de Chalon-sur-Saône. 
Néanmoins, Kollar n'est pas un traditionaliste. Sa pratique de la photographie est au contraire marquée par la modernité, par l'influence des nouveaux courants, de la Nouvelle Vision. Il est fasciné par les artefacts de la société industrielle. Il photographie les lignes des usines sidérurgiques, des machines, des ponts métalliques... Les techniques modernes et les machines ne sont pas absentes non plus chez les artisans qu'il a photographiés. Ses photographies laissent transpercer des évolutions dans le travail des artisans. Ce travail n'est pas figé dans la tradition. Le four du boulanger parisien est parcouru par le réseau des tuyaux qui l'alimentent et des cadrans de contrôle. Le pétrissage de la pâte est désormais fait à la machine. Les artisans ont dû intégrer dans la gamme de leur savoir-faire le maniement de ces machines. Ils ont adopté la mécanisation en l'adaptant à leurs besoins (Perrin, 2017). Le Boucher et sa camionnette font la tournée des hameaux d'un village de Charente ${ }^{18}$. L'acquisition de véhicules automobiles contribue à la mobilité, essentielle pour de nombreux corps de métiers où le travail est réalisé à l'extérieur, notamment les chantiers du bâtiment. Cette mobilité motorisée est aussi au cœur du métier des mariniers avec leur péniche. Par ailleurs, les artisans jouent un rôle important dans la diffusion de l'innovation qu'est l'automobile. François Kollar l'a bien compris en intégrant son Petit mécano de village dans la série Automobile. Cette industrie a besoin du réseau des artisans garagistes pour réparer et entretenir les voitures qu'elle produit. Les numéros de téléphone inscrits sur les façades des ateliers montrent comment ces artisans commencent aussi à s'emparer dans les années 1930 des nouveaux moyens de communication, à l'exemple de Léon Dupin, Le Mécanicien de village ${ }^{19}$. Son enseigne, comme les trois autres de mécanicien dans cette série du fer, donne aussi des précisions sur les prestations proposées. La devanture est un support de publicité pour les artisans (Perrin, 2012).

\subsection{Hors champ et oubliés?}

Certaines catégories de personnes apparaissent très peu sur ces photographies d'artisans, notamment les femmes, les membres de la famille et les jeunes apprentis. Faut-il y voir un oubli ou le reflet d'une absence bien réelle?

Les artisanes sont rares parmi les artisans photographiés mais elles le sont aussi dans l'artisanat. En effet, $80 \%$ des artisans immatriculés au registre des métiers dans les années 1930 sont des hommes. Les femmes sont même totalement absentes de certains secteurs d'activité comme le bâtiment. Les artisanes sont cantonnées dans l'habillement et les services d'entretien du linge ou de la personne. De ce point de vue, leur marginalité dans le reportage ne relève pas d'un oubli. Cependant, deux remarques peuvent être formulées pour nuancer ce constat. D'une part, l'absence des services, particulièrement la coiffure, accentue le cantonnement des artisanes dans le textile, qui plus est dans des savoir-faire traditionnels mais marginaux comme la dentellerie, avec ses variantes régionales, plutôt que la confection des vêtements. Ces images nourrissent le penchant régionaliste et traditionaliste du reportage. Elles reproduisent aussi les clichés sur les métiers d'aiguille réputés féminins. D'autre part, elles renvoient les femmes dans la sphère domestique. Elles sont représentées en train d'exécuter ces travaux de dentelle dans leurs intérieurs, au coin du feu ${ }^{20}$. L'artisane y est littéralement une femme au foyer. Elle exerce ici un métier qu'elle peut pratiquer chez elle. Alors que 
le travail artisanal est pour les hommes émancipateur et épanouissant, il contribue au contraire à maintenir les femmes à la maison.

Si les femmes artisans sont peu nombreuses dans le reportage, les femmes d'artisans le sont tout aussi peu, tout comme les enfants; ce qui pourrait a priori surprendre en considérant l'assimilation fréquente de l'artisanat au travail familial. Les familles sont bien présentes sur les péniches des bateliers, par exemple, mais parce qu'elles y vivent et elles n'y participent pas au travail. Trois critères sont habituellement retenus pour définir les entreprises familiales : la détention de la majorité du capital par la famille, l'emploi de membres de la famille, la transmission. Ils ne coïncident que partiellement avec les entreprises artisanales. La transmission semble se couler dans le mythe des artisans de père en fils mais dans les registres des métiers seulement un artisan sur cinq environ a repris l'activité de son père. Les artisans sont très majoritairement des ouvriers qui se mettent à leur compte en créant (ex-nihilo donc) leur entreprise, pas des héritiers. Il n'est donc pas si surprenant de ne pas trouver d'artisan travaillant avec son fils dans La France travaille. De même, si le travail familial est bien présent et important dans l'artisanat, il convient néanmoins de ne pas le surestimer, car il n'est pas généralisé. Les statistiques sur cet aspect sont peu nombreuses mais convergentes, et elles montrent que ce sont 20 à $25 \%$ des artisans qui emploient régulièrement des membres de leur famille, avec de fortes variations sectorielles. Il est beaucoup plus répandu dans l'alimentation, par exemple, où les épouses s'occupent de la partie commerciale, de la boutique. Il semble qu'il ait été plus fréquent chez les façonniers, et c'est bien plutôt dans la série sur les tisserands picards que des femmes sont représentées avec leur mari. Elles y apparaissent au côté de leur mari, dans un rôle accessoire, presque figuratif. En particulier, ce ne sont pas elles qui manient les métiers à tisser, comme sur les cartes postales d'ateliers familiaux de Saint-Étienne où les hommes travaillent et les femmes sont passives et presque jamais devant un métier alors que de nombreuses ouvrières en conduisaient (Dubesset, 1996). Les photographies des femmes au travail mettent en scène un ordre social dans lequel hommes et femmes ont des rôles assignés.

Enfin, l'apprentissage n'est présent que sur Le Bâtiment. Jeunes maçons réparant un mur ${ }^{21}$. L'image ne suggère pas l'acte de transmission du savoir lui-même; l'artisan maître d'apprentissage n'est pas présent, probablement parce que ces deux jeunes maçons sont suffisamment avancés dans leur formation pour être autonomes. En revanche, elle illustre bien une caractéristique majeure de l'apprentissage artisanal : il se fait « sur le tas ». C'est la réalisation et la répétition des gestes du métier qui permet d'en acquérir la maitrise. L'apprentissage est un enjeu important pour les organisations artisanales. C'est une compétence des chambres de métiers, précisée par la loi Walter-Paulin de 1937. En revanche, l'enjeu n'est pas le même pour les artisans eux-mêmes dont seulement un sur cinq forme des apprentis. La majorité des artisans ne sont pas acteurs de l'apprentissage. Il n'est donc pas si surprenant que le reportage trouve peu d'apprentis de l'artisanat à montrer. L'enseignement technique, et l'idée que le travail s'apprend, n'est pas pour autant absent de La France travaille qui présente à plusieurs reprises des écoles techniques. Ce choix de montrer l'apprentissage par les écoles plutôt que dans l'artisanat est en outre cohérent avec l'orientation prise par l'enseignement technique dans l'entre-deux-guerres sous l'impulsion de l'État qui privilégie la voie scolaire, avec la loi Astier de 1919 et le diplôme du CAP notamment (Lembré, 2013), au regard de laquelle l'apprentissage « sur le tas » apparait dépassé. 
François Kollar n'était ni sociologue ni journaliste et la France travaille n'est pas un reportage sur l'artisanat mais sur un terrain plus large qui comprend des artisans. Il ne propose donc pas une photographie fidèle de l'artisanat des années 1930 mais bien un regard sur les artisans. Certains métiers importants, particulièrement ceux du bâtiment, s'y trouvent ainsi sous-représentés alors que la place d'activités traditionnelles mais sur le déclin est exagérée. Plus globalement, les artisans servent souvent à incarner le versant traditionaliste et régionaliste de la publication que ce soit avec les «vieux métiers » ou par la place assignée aux femmes. Le reportage reproduit et véhicule des stéréotypes et des clichés qui en sont autant de limites. Néanmoins, et contrairement aux traditionalistes qui se retrouvèrent ensuite au pouvoir dans l'entourage du maréchal Pétain à Vichy, Kollar ne s'arrête pas à cette vision un peu désuète de l'artisanat. Sensible à la modernité industrielle, il en montre aussi les évolutions. Il présente, en somme, des artisans ancrés dans la tradition mais ouverts à la modernité. Surtout, en jouant sur les analogies visuelles, Kollar suggère bien la proximité persistante entre les artisans et le monde ouvrier. Ces photographies montrent que dans les années 1930, les artisans restent fondamentalement des ouvriers qui se sont mis à leur compte, des travailleurs indépendants qui exercent le plus souvent seuls. Ce n'est qu'après la guerre, au cours des Trente Glorieuses, que ces indépendants s'affirmèrent plus franchement en entrepreneurs, voire en petits patrons, et ce n'est pas avant les années 1970 que leur principal syndicat a cherché, avec succès, à siéger parmi les organisations représentatives des employeurs.

Huret Ch. (2014) « Images du travail/travail des images », Cahiers jungiens de psychanalyse, $\mathrm{n}^{\circ} 140$, p. 109-124.

\section{BIBLIOGRAPHIE}

Albera F. (2018) «Exposition : photographie arme de classe », 1895, n 86, p. 177-179.

Calame-Levert F., Decoux J. et Perroy A. (2012) Les Hommes de la mer : dans l'objectif de François Kollar, Paris, La Martinière.

Callens A.-C. (2020) «La représentation du travail dans les photographies publicitaires des éditions Paul-Martial », Images du travail, travail des images, $\mathrm{n}^{\circ} 8$.

Chevandier C. (2004) «At Work. Photographies sur les hommes et les femmes au travail», Vingtième Siècle. Revue d'histoire, $\mathrm{n}^{\circ}$ 84, p. 188-195.

Chevrier J.-F. (2016) «La France travaille : les vertus de l'illustration », dans François Kollar. Un Ouvrier du regard, La Martinière / Jeu de Paume, Paris.

Collectif (2016) François Kollar. Un ouvrier du regard, Catalogue d'exposition, Paris, La Martinière / Jeu de Paume. 
Crasset O. (2017) La Santé des artisans : de l'acharnement au travail au souci de soi, Rennes, Presses universitaires de Rennes.

Dancer-Mourès M. et Méaux D. (2014) Les Photographes et la commande industrielle autour des éditions Paul-Martial, Saint-Étienne, Musée d'art moderne et contemporain.

Daty G. (1933) L'Artisanat en Alsace, Colmar, Librairie Huffel.

Deilhes J. (2016) « Dominique Delpoux ou les visages du travail », Images du travail, travail des images, $\mathrm{n}^{\circ} 2$.

Dubesset M. (1996) «Femmes photographiées : images et représentations d'ouvrières et d'employées (Saint-Etienne, 1890-1940) », dans Peroni M. et Roux J., Le Travail photographié, Paris, CNRS éditions/Publications de l'Université de Saint-Étienne.

Denoyelle F. (1995) François Kollar. Le choix de l'esthétique, Paris, La Manufacture.

Friedmann G. (1956) Le Travail en miettes : Spécialisation et loisirs, Paris, Gallimard.

Guenne J. (1934) « Kollar ou le sens de la matière », L'Art vivant, janvier, p. 12.

Joschke C. (2020) « Introduction. Nouveaux regards sur la photographie ouvrière », Transbordeur. Photographie histoire société, $\mathrm{n}^{\circ}$ 4, p. 6-15.

Kessler M.-C. et Rousseau G. (dir.) (2018) Étienne Clémentel. Politique et action publique sous la Troisième République, Bruxelles, Peter Lang.

Kollar F. (1986) Les Hommes de l'État. Cheminots des années 30 (Le temps de la vapeur), Paris, La Vie du Rail.

Kollar F. (2010) (textes d'Édouard Lynch) Nous étions des paysans, Paris, La Martinière.

Lelieur A.-C. et Bachollet R. (1986) La France travaille. Regard sur le monde du travail à la veille du Front populaire, Paris, Éditions du Chêne.

Lembré S. (2013) L'École des producteurs. Aux origines de l'enseignement technique en France (1800-1940), Rennes, Presses universitaires de Rennes.

Morel G. (2001) « Du peuple au populisme », Études photographiques, n 9, p. 44-63.

Peroni M. et Roux J. (1996) Le Travail photographié, Paris, CNRS éditions/Publications de l’Université de Saint-Étienne.

Perrin C. (2007) Entre glorification et abandon. L'État et les artisans en France (1938-1970), CHEFF, Paris.

Perrin C. (2012) « Promouvoir son travail. La publicité des artisans en France au Xxe siècle », Recherche socialiste, 60-61, p. 87-104.

Perrin C. (2017) «L'artisanat, un ordre économique sans machine? Penser et repenser l'artisanat et la société française au Xxe siècle ", L'Homme et la Société, n 207, p. 185-209.

Perrin C. (2020) « Parole in storia : Artigiano/Artisan », Diacronie, Studi di storia contemporanea, 22, 2020, https://www.studistorici.com/2020/04/30/parole-in-storia-artisan-fr/

Rey A. (1992) Dictionnaire historique de la langue française, Paris, Le Robert (rééd. 2011).

Roegiers P. et Baqué D. (1989) François Kollar, Paris, Ministère de la Culture/Philippe Sers.

Schwint D. (2002) Le Savoir artisan. L'efficacité de la mètis, Paris, L'Harmattan, 2002.

Schwint D. (2005) « La routine dans le travail de l'artisan », Ethnologie française, n 35.

Sennett R. (2010) Ce que sait la main. La culture de l'artisanat, Paris, Albin Michel. 
Tailledet R. (1937) La Doctrine de classe de l'artisanat français, Paris, Institut national des métiers.

Terrenoire J.-P. (1985) « Images et sciences sociales; l'objet et l'outil », Revue française de sociologie, vol. 26, $\mathrm{n}^{\circ}$ 3, p. 509-527.

Zarca B. (1986) L'Artisanat français, du métier traditionnel au groupe social, Paris, Économica.

Zdatny S. (1999) Les Artisans en France au XXe siècle, Paris, Belin.

\section{NOTES}

1. Il signa le logo de la SNCF en 1938, par exemple. De son vrai nom Samuel Monod, il est le frère du biologiste et écrivain Théodore Monod.

2. Les liens en notes permettent d'accéder directement aux photographies citées.

3. Bibliothèque Forney (BF), RES ICO 75602 B887, https://bibliotheques-specialisees.paris.fr/ ark:/73873/pf0000581807/0188

4. BF, RES ICO $756010 \mathrm{P} 22$, https://bibliotheques-specialisees.paris.fr/ark:/73873/ pf0000618420/0021.

5. Désormais École nationale supérieure Louis Lumière.

6. «Proposition de loi sur l'artisanat », Le Photographe, nº 331, 5 février 1933, p. 38.

7. Id.

8. Le Photographe, $\mathrm{n}^{\circ} 379,1935$, p. 49.

9. Sous réserve de parvenir à le retrouver dans ce registre parmi les milliers d'artisans qui ont dû satisfaire à la même démarche en même temps que lui à Paris.

10. Sur l'émergence d'une esthétique industrielle, voir Dancer-Mourès et Méaux (2014).

11. François Kollar a également fait des images pour les éditions Paul-Martial (Callens, 2020)

12. BF, RES ICO 75609 O64, https://bibliotheques-specialisees.paris.fr/ark:/73873/ pf0000618395/0058?

13. Au cours de ses vingt-et-une années d'existence (1996-2017), la revue Études photographiques a témoigné de bien peu d'intérêt pour François Kollar qui ne s'y trouve mentionné qu'une seule fois.

14. BF, RES ICO 7560 9094, https://bibliotheques-specialisees.paris.fr/ark:/73873/ pf0000618395/0086

15. BF, RES ICO 7560 6 C96, https://bibliotheques-specialisees.paris.fr/ark:/73873/ pf0000617395/0007

16. https://bibliotheques-specialisees.paris.fr/ark:/73873/pf0000618436/0085

17. BF, RES ICO $7560 \quad 8 \mathrm{~L} 62$, https://bibliotheques-specialisees.paris.fr/ark:/73873/ pf0000618386/0058

18. BF, RES ICO $7560 \quad 8$ K71, https://bibliotheques-specialisees.paris.fr/ark:/73873/ pf0000618362/0070

19. BF, RES ICO 7560 2 B889, https://bibliotheques-specialisees.paris.fr/ark:/73873/ pf0000581807/0189

20. BF, RES ICO $756011 \mathrm{~T} 64$, https://bibliotheques-specialisees.paris.fr/ark:/73873/ pf0000618440/0061

21. BF, RES ICO 7560 13 R1, https://bibliotheques-specialisees.paris.fr/ark:/73873/ pf0000618436/0001 


\section{RÉSUMÉS}

Le photographe François Kollar accède à la notoriété avec le reportage La France travaille publié par les éditions Horizons de France entre 1931 et 1934. Ces images, conservées aujourd'hui par la bibliothèque Forney, sont un document pour écrire l'histoire du travail. L'article s'intéresse aux artisans dans ce reportage. Il se demande dans quelle mesure ces photographies composent une archive du travail des artisans. Il les confronte tout d'abord à nos connaissances sur l'artisanat des années 1930, un groupe socioprofessionnel qui s'organise dans l'entre-deux-guerres et dont Kollar est proche. Puis, il analyse ce que Kollar montre de ces travailleurs, de leur travail, de ses opérations, gestes, postures et savoir-faire, mais aussi ce qu'il a laissé hors champ.

Photographer François Kollar gained notoriety with the reportage La France travaille published by Horizons de France Publishing between 1931 and 1934. These images, today in the Forney library, are a document to write the history of the work. The article focuses on artisans in this reportage. It wonders to what extent these photographs constitute an archive of the craftsmen's work. It first confronts them with our knowledge of the crafts of the 1930s, a socio-professional group that was organized itself in the interwar period and to which Kollar is close. Then, it analyses what Kollar shows about these workers, their work, its operations, gestures, postures and knowhow, but also what he left off-camera.

\section{INDEX}

Mots-clés : photographie, François Kollar, artisan, artisanat, travail

Keywords : photography, François Kollar, craftsman, artisanat, work

\section{AUTEUR \\ CÉDRIC PERRIN}

Cédric Perrin, agrégé et docteur en Histoire, enseigne au lycée Grandmont à Tours et à l'université Évry Paris-Saclay. Il est chercheur rattaché à l'IDHE.S Évry. Ses travaux de recherche portent sur l'histoire politique et économique des artisans et sur l'histoire des techniques. Il a notamment publié Entre glorification et abandon. L'État et les artisans en France (1938-1970) (CHEFF, Paris, 2007) et co-dirigé « Le temps des artisans. Permanences et mutations. », Marché \& organisations, $\mathrm{n}^{\circ} 24$ (2015). 